\title{
Interrupción inmediata versus manejo expectante luego de rotura prematura de membranas cercano al término del embarazo (ensayo PPROMT): un ensayo controlado randomizado (1)
}

Morris JM, Roberts CL, Bowen JR, Patterson JA, Bond DM, Algert CS, Thornton JG, Crowther CA; PPROMT Collaboration. Immediate delivery compared with expectant management after preterm pre-labour rupture of the membranes close to term (PPROMT trial): A randomised controlled trial.Lancet 2016;387(10017):444-52.

Análisis Crítico: Rafael Valdés V., Jorge A Carvajal C. PhD.

Unidad de Medicina Materno-Fetal, División de Obstetricia y Ginecología, Escuela de Medicina, Pontificia Universidad Católica de Chile.

\section{RESUMEN}

Introducción: La rotura de membranas cercanas a término, se asocia con un mayor riesgo de infección neonatal, pero la interrupción inmediata se asocia a un riesgo mayor de patologías asociadas a la prematuridad. El balance de riesgos aún no está claro. El objetivo fue establecer si el parto inmediato en los embarazos únicos con rotura de membranas cercanas a término reduce la infección neonatal sin aumentar otra morbilidad. Método: EI PPROMT fue un ensayo controlado aleatorio multicéntrico realizado en 65 centros en 11 países. Fueron incluidas mujeres mayores de 16 años, con embarazos únicos y con rotura de membranas antes del inicio del trabajo de parto, entre las 34 semanas y 36 semanas y 6 días, en ausencia de signos de infección. Fueron asignadas al azar (1:1) por un programa de aleatorización generada por computador con tamaños de bloque variables, estratificados por centro, en interrupción inmediata o manejo expectante. El resultado primario fue la incidencia de sepsis neonatal. Los resultados secundarios neonatales incluyeron un indicador de morbilidad y mortalidad neonatal compuesta (es decir, sepsis, ventilación mecánica $\geq 24$ horas, muerte fetal o muerte neonatal); síndrome de dificultad respiratoria; uso de ventilación mecánica y la duración de hospitalización en una unidad de cuidados intensivos neonatales.
Los resultados maternos secundarios incluyeron hemorragia antes del parto o durante el parto, fiebre intraparto, tratamiento postparto con antibióticos, y el modo de parto. Las mujeres y los médicos no podían ser enmascarados, pero aquellos que conocieron el resultado primario, fueron ciegos a la asignación de grupos. Los análisis fueron por intención de tratar. Este ensayo fue registrado en el Registro Internacional de Ensayos Clínicos. Resultados: Entre el 28 de mayo de 2004 y 30 de junio de 2013, 1839 mujeres fueron reclutadas y asignadas al azar: 924 al grupo interrupción inmediata y 915 al grupo de manejo expectante. Una de las mujeres en el grupo de parto inmediato y tres en el grupo expectante, fueron excluidos de los análisis primarios. La sepsis neonatal ocurrió en $23(2 \%)$ de los 923 recién nacidos cuyas madres fueron asignadas a interrupción inmediata y 29 (3\%) de 912 neonatos de madres asignadas a conducta expectante (riesgo relativo [RR] 0,8; IC95\% 0,5 $-1,3 ; p=0,37)$. El resultado secundario compuesto de morbilidad y mortalidad neonatal se presentó en $73(8 \%)$ de 923 neonatos de madres asignadas a interrupción inmediata y 61 (7\%) de 911 neonatos de madres asignadas a conducta expectante (RR $1,2 ; \mathrm{IC} 95 \% 0,9-1,6 ; p=0,32)$. Sin embargo, en el grupo de interrupción inmediata, los recién nacidos presentaron un aumento en las tasas de dificultad respiratoria (76 [8\%] de 919 vs 47 [5\%] de 910, RR 
1,6; IC95\% 1,1-2,30; $p=0,008)$, uso de ventilación mecánica (114 [12\%] de 923 frente a $83[9 \%]$ de 912, RR 1,4; IC95\% 1,0-1,8; p=0,02) y mayor tiempo de estadía en unidad de cuidados intensivos neonatales (mediana de 4,0 días [IQR 0,0-10,0] vs $2,0$ días $[0,0-7,0] ; p<0,0001)$ en comparación con los recién nacidos de madres del grupo de manejo expectante. Las pacientes del grupo expectante, tuvieron mayor riesgo de hemorragia antes del parto o durante el parto (RR 0,6; IC95\% 0,4-0,9), fiebre intraparto (RR 0,4; IC95\% 0,2-0,9), y uso de antibióticos después del parto (RR 0,8; IC95\% 0,7-1,0), y estadía hospitalaria más prolongada $(p<0,0001)$, pero un menor riesgo de cesárea (RR 1,4; IC $95 \%$ 1,2-1,7). Conclusiones: En ausencia de signos manifiestos de infección o compromiso fetal, una política de manejo expectante se debe seguir en mujeres embarazadas que se presentan con rotura de membranas cercanas al término, con vigilancia apropiada del bienestar materno y fetal.

\section{ANÁLISIS DE LA INVESTIGACIÓN}

\section{A. Relevancia clínica de la investigación}

Rotura prematura de las membranas (rotura de las membranas antes del inicio del trabajo de parto) se produce en el $20 \%$ de todos los embarazos y en el $40 \%$ de todos los partos prematuros. Al término, existe una sólida evidencia de que la interrupción inmediata se asocia a una menor incidencia de infección materna y mayor satisfacción materna en comparación con el manejo expectante, sin riesgos concomitantes en la morbilidad o la mortalidad perinatal. Por el contrario, el manejo óptimo de los embarazos con rotura prematura de membranas antes de las 37 semanas no es claro (2). La práctica clínica en estas circunstancias varía sustancialmente a nivel internacional, sobre todo en embarazos cercanos al término (es decir, más allá de las 34 semanas de gestación). La interrupción inmediata es recomendada por el Colegio Americano y de Inglaterra de Ginecología y Obstetricia, con conclusiones como "en embarazos de 34 semanas o más, se recomienda interrupción inmediata de todas las mujeres con rotura de membranas". Estos grupos reconocen que este tipo de conclusiones están basadas en evidencia científica limitada e inconsistente (3-5). Por lo tanto, a diferencia de la rotura prematura de membranas a término, la rotura prematura pretérmino de membranas sigue planteando un dilema clínico. Los riesgos del manejo espectante, tales como desprendimiento de la placenta, infección ascendente, sufrimiento fetal durante el parto y prolapso del cordón, necesitan equilibrarse con el consiguiente riesgo de prematuridad iatrogénica de la interrupción inmediata (4-5). Cuando el embarazo alcanza la viabilidad o está cerca de ella
(23-30 semanas de gestación) y en ausencia de infección establecida o compromiso materno o fetal, hay unanimidad en que el tratamiento expectante es deseable, porque los fetos prematuros nacidos antes de las 30 semanas tienen un mayor riesgo de muerte neonatal y morbilidad grave (hemorragia intraventricular, enfermedad de membrana hialina, y enterocolitis necrotizante). Sin embargo, estos riesgos son muchos menores cuando la edad gestacional alcanzada es cercana al término. Recomendaciones para interrupción inmediata después de la rotura de membranas cercanas a término, necesitan estar respaldada por sólida evidencia clínica porque incluso la prematuridad, probablemente con morbilidad leve, se puede asociar con complicaciones neonatales, tanto a corto como a largo plazo, lo que llevaría a un aumento en el gasto en salud para el país.

\section{B. El estudio (1)}

Diseño: Estudio multicéntrico, randomizado y controlado que compara interrupción inmediata con manejo expectante (control) en mujeres con embarazos únicos, de 65 maternidades, en 11 países.

Pacientes: Mujeres mayores de 16 años, embarazo únicos y clínicamente con sospecha rotura de membranas entre las 34 semanas y 36 semanas y 6 días de gestación. Las mujeres que se presentaron con rotura de membranas previo a estas semanas de embarazo, fueron elegibles al llegar a las 34 semanas de gestación.

Intervención: Las mujeres en el grupo de interrupción inmediata debían ser interrumpidas tan cerca de la aleatorización como sea posible y preferentemente dentro de las primeras 24 horas. El modo de parto fue según indicaciones obstétricas habituales.

Comparación: Manejo expectante hasta las 37 semanas de embarazo. Debido a que no existe un tratamiento estándar en lo que respecta al manejo de la paciente después de la rotura prematura de las membranas, las mujeres fueron manejados de acuerdo a normativas locales. A lo largo del periodo de reclutamiento, los antibióticos se consideraron como parte del tratamiento, y éstos fueron prescritos de acuerdo con los protocolos locales. Se solicitó histología placentaria. Sólo se evaluaron los cultivos de muestras vaginales tomadas entre la rotura prematura de las membranas y la asignación al azar, y los resultados de la flora vaginal normal y lactobacilos se clasificaron como negativos. Todas las demás características de los pacientes fueron informadas por los participantes al ingresar al estudio o recogidas de las historias clínicas.

Resultados Medidos: Resultado primario: Incidencia de sepsis neonatal, ya sea definitiva o probable, establecido por revisión exhaustiva de los 
datos neonatales por un comité central de adjudicación enmascarado a la asignación del tratamiento. Resultados secundarios: Neonatales: Morbilidad neonatal compuesta e indicador de mortalidad (sepsis, ventilación mecánica $\geq 24 \mathrm{hrs}$, muerte fetal o muerte neonatal); síndrome de dificultad respiratoria; mortalidad perinatal; neumonia; ventilación mecánica (ventilación intermitente de presión positiva, presión positiva continua de las vías respiratorias, o ventilación de alta frecuencia), ventilación mecánica durante al menos 24 hrs; tiempo en una unidad de cuidados intensivos neonatales, peso de nacimiento; pequeños para la edad gestacional (< percentil 10); Apgar menor a 7 a los 5 minutos; antibióticos en las primeras 48 h; punción lumbar; compromiso circulatorio con necesidad de línea arterial, bolo, o soporte inotrópico; lactancia materna al alta. Los resultados neonatales se obtuvieron de los diagnósticos informados por el médico a cargo de los registros y recogidos durante 28 días o hasta el alta. Resultados maternos secundarios: hemorragia antes del parto o durante el parto, trombosis previo o postparto, prolapso del cordón, tratamiento con antibióticos después del parto, fiebre intraparto $\left(\geq 38,5^{\circ} \mathrm{C}\right)$, hemorragia postparto $(>1000 \mathrm{ml})$, tipo de parto, inicio del parto, y duración de la hospitalización (días totales desde la aleatorización hasta el parto). La corioamnionitis fue un criterio de exclusión del ingreso al ensayo, pero este resultado secundario fue evaluado en las pacientes con manejo expectante.

\section{Análisis crítico}

Validez interna: Estudio con descripción apropiada de los criterios de inclusión y exclusión, multicéntrico, randomizado, ciego, con ocultamiento de la secuencia de randomización, similitud de pacientes en ambos grupos, con seguimiento completo y análisis por intención de tratar. Se respetó el cálculo del tamaño muestral propuesto antes de iniciar el estudio (1839 pacientes) para detectar una reducción desde $5 \%$ de infección neonatal en el manejo expectante vs. $2,5 \%$ en el grupo de interrupción inmediata.

Validez externa: El estudio es aplicable a nuestra población, y las posibilidades de ambos tipos de manejos son factibles de realizar en nuestro país.

Conclusiones: Estudio con bajo riesgo de sesgo y metodología adecuada, por lo que sus resultados deben ser considerados como válidos. El estudio demostró que el manejo expectante en pacientes con rotura prematura de membranas cercano al término, es una conducta adecuada. Este manejo disminuiría la morbilidad neonatal, aumentando levemente el riesgo de hemorragia (intraparto y postparto), además de disminuir la tasa de cesáreas, sin aumentar el riesgo de infección materna y neonatal. Este estudio nos proporciona información relevante y que podría cambiar el manejo actual de las roturas prematuras cercanas al término en las maternidades de nuestro país. Creemos posible y necesario replicar este protocolo, y así poder reafirmar lo demostrado en este estudio, demostrando localmente los beneficios del manejo expectante vs. interrupción inmediata en rotura de membranas cercano al término del embarazo.

\section{REFERENCIAS}

1. Morris JM, Roberts CL, Bowen JR, Patterson JA, Bond DM, Algert CS, Thornton JG, Crowther CA; PPROMT Collaboration. Immediate delivery compared with expectant management after preterm pre-labour rupture of the membranes close to term (PPROMT trial): A randomised controlled trial. Lancet. 2016;387(10017):444-52.

2. Hannah ME, Ohlsson A, Farine D, et al. Induction of labor compared with expectant management for prelabor rupture of the membranes at term. TERMPROM Study Group. N Engl J Med 1996; 334: 1005-10.

3. Buchanan SL, Crowther CA, Levett KM, Middleton P, Morris J. Planned early birth versus expectant management for women with preterm prelabour rupture of membranes prior to 37 weeks' gestation for improving pregnancy outcome. Cochrane Database Syst Rev 2010; 3: CD004735.

4. Van der Ham DP, Vijgen SM, Nijhuis JG, et al. Induction of labor versus expectant management in women with preterm prelabor rupture of membranes between 34 and 37 weeks: a randomised controlled trial. PLoS Med 2012; 9: e1001208.

5. Van der Ham DP, van der Heyden JL, Opmeer BC, et al. Management of late-preterm premature rupture of membranes: the PPROMEXIL-2 trial. Am J Obstet Gynecol 2012; 207: 276, e1-10. 